\title{
DISEÑO E IMPLEMENTACIÓN DE LABORATORIO DE TOPOGRAFía PARA LAS CARRERAS DE LA FACULTAD DE EDUCACIÓN TÉCNICA PARA EL DESARROLLO (FETD), DE LA UNIVERSIDAD CATÓLICA DE SANTIAGO DE GUAYAQUIL DESIGN AND IMPLEMENTATION OF TOPOGRAPHY LABORATORY FOR THE FACULTY OF TECHNICAL EDUCATION FOR DEVELOPMENT (FETD), IN UNIVERSIDAD CATOLICA DE SANTIAGO DE GUAYAQUIL
}

\section{JOSÉ RICARDO RIVAS BARZOLA', ALBERTO PEÑALVER², JUAN ALFREDO PADILLA CERDAN ${ }^{3}$}

\footnotetext{
1 Universidad Católica de Santiago de Guayaquil. jose.rivas02@cu.ucsg.edu.ec

2 Universidad Católica de Santiago de Guayaquil. alberto.penalver01@cu.ucsg.edu.ec

3 Universidad Católica de Santiago de Guayaquil. alfredpadilla_17@hotmail.com
}

RESUMEN

El presente artículo identifica la incidencia del componente práctico en las materias de Topografía, Construcciones Rurales, Suelos y Drenajes para el desarrollo de habilidades y destrezas de los estudiantes, mediante el manejo de equipos topográficos. Esto, con la finalidad de diseñar e implementar un laboratorio de topografía a través de un proyecto de investigación no experimental con enfoque cualitativo y cuantitativo. Se analizó como variable independiente el componente práctico, y como variable dependiente las habilidades y destrezas de los estudiantes durante el uso de equipos topográficos. Para el levantamiento de información fueron aplicadas las siguientes técnicas: observación, encuestas, entrevistas, análisis de documentos, test, examen práctico. Los actores involucrados en la recogida de información fueron: estudiantes que estaban cursando la materia de Topografía y aquellos que ya la habían cursado; docentes con materias relacionadas al uso de equipos topográficos; y expertos en el tema. Una vez recopilados los documentos y analizada la información, se generó una base de datos con Microsoft Excel, para determinar porcentualmente los resultados. Estos demostraron que el tiempo dedicado presencialmente a las asignaturas relacionadas con la Topografía, ocupa el $6.12 \%$ del total de horas curriculares; $y$ los syllabus de las asignaturas no permiten establecer el tiempo de las prácticas. El test aplicado a estudiantes evidenció que no poseen habilidades y destrezas en el manejo instrumental de equipos topográficos. Por lo tanto, se concluye que la falta de un laboratorio de Topografía y medios de enseñanza necesarios impide a los estudiantes ser competitivos en el mercado laboral.

PALABRAS CLAVE: Ingeniería Agropecuaria, topografía, geomática, habilidades y destrezas.

\section{ABSTRACT}

The present research identifies the incidence of the practical component in the subjects of Topography, Rural Constructions, Soils and Drainage for the development of skills and abilities of the students through the management of topographic equipment, with the purpose of designing and implementing a Topography Laboratory through a non-experimental research project with a qualitative and quantitative approach. We analyzed an independent variable referring to the practical component, and a dependent variable referring to the skills and abilities of the students during the use of topographic equipment. For the collection of information, techniques such as observation, surveys, interviews, document analysis, tests, practical test, were applied to students studying the subject of Topography and those who already did; Teachers with subjects related to the use of topographic equipment and experts in the subject. Once the documents were collected and the information analyzed, a database was generated with Microsoft Excel, to determine the results percentage; Which show that the time dedicated to the subjects related to Topography occupies $6.12 \%$ of the total curricular hours; The syllabus of the subjects do not allow to establish the time of the practices, and the test applied to students evidences that they do not possess abilities and skills in the instrumental management of topographic equipment. Therefore, the lack of a Topography Laboratory and necessary teaching aids prevents students from being competitive in the labor market.

KEYWORDS: Agricultural Engineering, surveying, geomatics, abilities and skills. 
INTRODUCCIÓN

En Ecuador, el gobierno a partir del año 2008 hasta el 2017, determinó que la educación superior es un sistema que responde al interés público sin fines de lucro; involucrándose en un proceso de mejora en todos sus estamentos. Es así que, en el aspecto académico, las Instituciones de Educación Superior (IES) deben cumplir una serie de requisitos previstos en el proceso de evaluación y acreditación, a través de la medición de las habilidades y destrezas desarrolladas por los estudiantes; evidenciando los conocimientos recibidos en el aula. Ello se convierte en una alternativa que permitirá al futuro profesional desempeñarse adecuadamente en su trabajo.

Una forma de incentivar el aprendizaje es mediante la aplicación de adecuadas metodologías de enseñanza-aprendizaje que presentan las asignaturas en sus syllabus. Las IES en el Ecuador están sujetas a un control exhaustivo por parte de la Secretaría de Educación Superior, Ciencia, Tecnología e Innovación (SENESCYT) y el Consejo de Evaluación, Acreditación y Aseguramiento de la Calidad de la Educación Superior (CEAACES); a partir del cual, la academia es evaluada midiendo los resultados del aprendizaje de los estudiantes y profesionales, en donde se evidencia el nivel del desarrollo de habilidades y destrezas.

En un estudio realizado por Ramírez y Martínez (2010), se define al reconocimiento y la modificación de las concepciones sobre el aprendizaje y la práctica profesional, como una posibilidad de impedir la continuidad de prácticas de enseñanzas identificadas por la historia educativa personal y la herencia socio cultural (paradigma tradicional). Los docentes, responsables de hacer cumplir las metas propuestas son uno de los pilares más importantes en el proceso de enseñanza-aprendizaje, ya que imparten sus conocimientos y realizan la planificación de las prácticas.

Las IES, de acuerdo al reglamento de Régimen Académico, en su artículo 15 inciso 2, declaran: "el desarrollo de experiencias de aplicación de los aprendizajes, mediante actividades académicas desarrolladas en escenarios experimentales o en laboratorios" (CES, 2014). Durante la investigación que sirvió de base al presente artículo fue necesaria la determinación de las habilidades prácticas de los estudiantes, haciendo uso de los equipos topográficos facilitados por los investigadores. Como resultado fue identificada la necesidad del establecimiento de un laboratorio de topo- grafía en la Facultad de Educación Técnica para el Desarrollo (FETD) con la finalidad de mejorar las habilidades en los estudiantes.

El objetivo de la investigación desarrollada fue demostrar la incidencia del componente práctico de las materias de Topografía, Construcciones Rurales, Suelos y Drenajes, en el desarrollo de habilidades y destrezas de los estudiantes de la FETD para el manejo de los equipos respectivos.

\section{METODOLOGÍA}

A continuación, se muestra la metodología utilizada:

A. Sistema de VARiables. Las variables que fueron medidas permitieron identificar la necesidad del establecimiento de un laboratorio de topografía. A continuación se muestran detalles:

- Variable independiente: Componente práctico.

- Categorías: Aplicación, formación general y perfil de egresado.

- Indicadores: número de créditos, número de asignaturas, número de horas prácticas.

- Técnicas: Observación, encuestas, entrevistas.

- Variable independiente: habilidades y destrezas.

- Categorías: habilidad, aptitud e idoneidad.

- Indicador: tiempo de realización de una tarea bien ejecutada.

- Técnicas: observación, encuestas, entrevistas, test, examen práctico.

\section{BREVE RESUMEN DE LAS TÉCNICAS E INSTRUMENTOS UTILIZADOS} PARA EL LEVANTAMIENTO DE LA INFORMACIÓN:

- Encuesta: Aplicada a profesores y estudiantes para indagar las principales opiniones y percepciones sobre los aspectos en estudio.

- Entrevista: Aplicada a expertos del área con el fin de recabar información más detallada sobre la incidencia de un laboratorio de topografía en la formación integral de los estudiantes.

- Análisis de documentos: Aplicado en el análisis de los syllabus de las materias involucradas con la finalidad de contrastar las clases programadas con las prácticas realizadas en laboratorio, de manera que permita mantener, modifi- 
car y/o temporizar las clases teóricas con las prácticas declaradas en los syllabus.

- Examen práctico: equipo topográfico armado completo (GPS, base, antena, y otros), cronómetro y test para evaluación los estudiantes seleccionados aleatoriamente, cámara fotográfica.

CRITERIOS DE INCLUSIÓN Y EXCLUSIÓN DE LAS MUESTRAS

\begin{tabular}{|c|c|}
\hline CRITERIOS DE INCLUSIÓN & CRITERIOS DE EXCLUSIÓN \\
\hline $\begin{array}{l}\text { Selección aleatoria de estudiantes } \\
\text { que estaban cursando la materia de } \\
\text { Topografía y los estudiantes que ya la } \\
\text { habían cursado. } \\
\text { Docentes de materias relacionadas y } \\
\text { que hagan uso de equipos topográficos } \\
\text { como: Topografía, Construcciones } \\
\text { Rurales, Edafología y Conservación de } \\
\text { Suelos, Riego y Drenaje. }\end{array}$ & $\begin{array}{l}\text { Estudiantes que aún no } \\
\text { han visto la materia de } \\
\text { Topografía. } \\
\text { Docentes de las demás } \\
\text { carreras de la FETD, } \\
\text { cuyas materias no están } \\
\text { relacionadas con la } \\
\text { Topografía. }\end{array}$ \\
\hline
\end{tabular}

b. Población/Muestra/Muestreo: A continuación, en la Tabla 1, se detalla el tamaño del grupo y de la muestra por grupo de individuos, el tipo de muestreo, y las técnicas para la recolección de información.

\begin{tabular}{|c|c|c|c|c|}
\hline $\begin{array}{l}\text { GRUPO } \\
\text { INDIVIDUO }\end{array}$ & $\begin{array}{l}\text { TAMAÑO } \\
\text { GRUPO (N) }\end{array}$ & $\begin{array}{l}\text { TAMAÑO } \\
\text { MUESTRA (n) }\end{array}$ & $\begin{array}{l}\text { TIPO } \\
\text { MUESTREO }\end{array}$ & $\begin{array}{l}\text { MÉTODO } \\
\text { TÉCNICA }\end{array}$ \\
\hline Estudiantes & 51 & 49 & aleatorio & encuesta, test \\
\hline Docentes & 4 & 4 & intencional & encuesta \\
\hline $\begin{array}{l}\text { Expertos } \\
\text { (nacional e } \\
\text { internacional) }\end{array}$ & 2 & 2 & intencional & encuesta \\
\hline
\end{tabular}

c. Tipo/Diseño de Investigación: Mixto (cuantitativo/cualitativo), No experimental. En cuanto a la Tabla 2, se muestra la fórmula aplicada para definir el tamaño de la muestra.

\section{TABLA 2. CÁLCULO DEL TAMAÑo MUESTRAL}

\begin{tabular}{|c|c|c|}
\hline ERROR & $3.0 \%$ & \\
\hline tAMAÑ̃o poblacióN & 51 & $N *\left(\alpha_{c} * 0,5\right)^{2}$ \\
\hline $\begin{array}{l}\text { NIVEL DE CONFIANZA } \\
\text { TAMAÑNO DE LA MUESTRA }\end{array}$ & $\begin{array}{l}95 \% \\
49\end{array}$ & $1+\left(e^{2} *(N-1)\right)$ \\
\hline
\end{tabular}

Una vez recopilados los datos mediante las técnicas mencionadas anteriormente, se procedió al análisis a través del programa utilitario informático Excel. Ello permitió identificar, de manera porcentual, las respuestas obtenidas partiendo de una línea base (generación de base de datos).
RESULTADOS ALCANZADOS

NIVEL DE PERTINENCIA DEL COMPONENTE PRÁCTICO DE LAS MATERIAS TOPOGRAFÍA, CONSTRUCCIONES RURALES, EDAFOLOGÍA Y CONSERVACIÓN DE SUELOS, RIEGO Y DRENAJES, REALIZANDO UN ANÁLISIS DE SYLLABUS Y ENCUESTAS A ESTUDIANTES Y DOCENTES

La revisión de los documentos de planificación del proceso de enseñanza y aprendizaje de la actual oferta de carreras agropecuarias de la FETD de la Universidad Católica de Santiago de Guayaquil (UCSC) (Ingeniería Agropecuaria, Ingeniería Agroindustrial, Medicina Veterinaria y Zootecnia), evidenció que las competencias profesionales relacionadas con la topografía solamente están presentes en la carrera de Ingeniería Agropecuaria.

En las entrevistas complementarias realizadas a los docentes de las asignaturas de Matemáticas, Física y Dibujo, estos consideran que imparten los fundamentos de los conocimientos que se aplican en la asignatura de Topografía:

- Matemáticas: geometría, trigonometría, derivación e integrales para cálculo de áreas, otros.

- Física: óptica, lentes, niveles, presión atmosférica, laser, telemetría, electromagnetismo, trasmisión/recepción de radio, otros.

- Dibujo: planos, AutoCAD, áreas, simbología.

La Tabla 3 indica las asignaturas y horas dedicadas a la formación de habilidades y destrezas en áreas de conocimientos relacionados con la Topografía, de acuerdo al criterio adoptado en esta investigación para definir las asignaturas de relación directa con la representación de la superficie terrestre.

\begin{tabular}{|c|c|c|c|c|}
\hline CICLO & ASIGNATURA & $\begin{array}{l}\text { HORAS/ } \\
\text { SEMANA }\end{array}$ & $\begin{array}{l}\text { TOTAL/ } \\
\text { SEMESTRE }\end{array}$ & $\%$ \\
\hline III & Topografía & 3 & 48 & $1.22 \%$ \\
\hline IV & Sistemas de información geográfica & 3 & 48 & $1.22 \%$ \\
\hline V & Construcciones rurales & 3 & 48 & $1.22 \%$ \\
\hline V & Edafología y conservación de suelos & 3 & 48 & $1.22 \%$ \\
\hline \multirow[t]{3}{*}{ VI } & Riegos y drenajes & 3 & 48 & $1.22 \%$ \\
\hline & Subtotal Horas & & 240 & $6.12 \%$ \\
\hline & Total Horas Presenciales & & 3920 & $100 \%$ \\
\hline
\end{tabular}

Nota: Tomado de FETD- UCSG (2012).

El tiempo dedicado presencialmente a las asignaturas directamente relacionadas con aplicaciones para la representación de la super- 
ficie terrestre ocupa el $6.12 \%$ del total de horas presenciales curriculares. Los syllabus de las asignaturas involucradas en el proyecto no permiten establecer el tiempo de las prácticas y los resultados de aprendizajes planificados para el desarrollo de destrezas instrumentales y aplicaciones de los conocimientos topográficos.

ENCUESTA DE OPINIÓN APLICADAS A LOS ESTUDIANTES

En la Tabla 4 se muestran los resultados obtenidos de las encuestas de opinión aplicada a los estudiantes.

\section{TABLA 4. RESUMEN DE ENCUESTAS APLICADAS A ESTUDIANTES DE} TOPOGRAFÍA III CICLO, SEMESTRE A2015

\begin{tabular}{|c|c|c|c|c|c|}
\hline RESUMEN & & & & & INDICADORES \\
\hline PREGUNTAS & 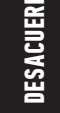 & 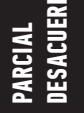 & 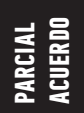 & $\begin{array}{l}\text { 号 } \\
\text { 兽 }\end{array}$ & SUMATORIA \\
\hline $\begin{array}{l}\text { 1. Piensa ud. que la } \\
\text { actividad práctica en la } \\
\text { materia de topografía } \\
\text { es primordial para el } \\
\text { desarrollo de ésta a } \\
\text { plenitud. }\end{array}$ & $0 \%$ & $0 \%$ & $40 \%$ & $60 \%$ & $100 \%$ \\
\hline $\begin{array}{l}\text { 2. Un laboratorio de } \\
\text { topografía puede servir } \\
\text { para otras materias de la } \\
\text { carrera. }\end{array}$ & $0 \%$ & $21 \%$ & $35 \%$ & $44 \%$ & $100 \%$ \\
\hline $\begin{array}{l}\text { 3. Tener conocimientos } \\
\text { hoy en día sobre GPS y } \\
\text { Estaciones Totales en } \\
\text { la rama de ingeniería } \\
\text { agrícola y/o agropecuaria } \\
\text { es necesario? }\end{array}$ & $0 \%$ & $24 \%$ & $31 \%$ & $45 \%$ & $100 \%$ \\
\hline $\begin{array}{l}\text { 4. Cree ud. que la parte } \\
\text { teórica debe ser superior } \\
\text { que la práctica en la } \\
\text { materia de topografía. }\end{array}$ & $0 \%$ & $21 \%$ & $29 \%$ & $41 \%$ & $100 \%$ \\
\hline $\begin{array}{l}\text { 5. Cree Ud. que la parte } \\
\text { práctica debe ser superior } \\
\text { que la teórica en la } \\
\text { materia de topografía. }\end{array}$ & $0 \%$ & $21 \%$ & $35 \%$ & $44 \%$ & $100 \%$ \\
\hline $\begin{array}{l}\text { 6. Piensa Ud. que los } \\
\text { conocimientos que pueda } \\
\text { adquirir en la materia de } \\
\text { topografía, los ponga en } \\
\text { práctica en el campo real. }\end{array}$ & $0 \%$ & $23 \%$ & $33 \%$ & $44 \%$ & $100 \%$ \\
\hline
\end{tabular}

De acuerdo con los resultados de la encuesta se puede afirmar que:

1. El componente práctico en la asignatura de Topografía es necesario para el desarrollo de habilidades y destrezas en los estudiantes de la carrera de Ingeniería Agropecuaria.

2. El laboratorio de topografía servirá a otras asignaturas de la carrera cuyos objetos de estudio sean similares.
3. Es necesario tener conocimiento del manejo instrumental de equipos Global Positioning System (CPS) y Estaciones Totales de Topografía.

4. El componente práctico en la asignatura de Topografía debe tener un mayor número de horas que el componente teórico.

5. Los conocimientos, habilidades y destrezas adquiridas en la asignatura de Topografía los pondrá en la práctica del ejercicio profesional de la carrera.

Los resultados de la encuesta aplicada a los 33 estudiantes que ya habían cursado la materia de Topografía, se indican en la Tabla 5.

TABLA 5. RESUMEN DE ENCUESTAS APLICADAS A ESTUDIANTES QUE YA HAN CURSADO LA MATERIA DE TOPOGRAFÍA

RESUMEN
PREGUNTAS

$\begin{aligned} & \text { 5. El establecimiento } \\ & \text { de un laboratorio de }\end{aligned}$
topografía sería una
$\begin{aligned} & \text { respuesta para mejorar } \\ & \text { la enseñanza de esta }\end{aligned}$
materia.

6. Considera ud. que

recibió en la materia de

topografía los conceptos $\quad 12 \% \quad 30 \% \quad 58 \% \quad 0 \% \quad 100 \%$

y aplicaciones de

programas y equipos.

Las opiniones de los estudiantes encuestados indican que:

1. El componente práctico en la asignatura de Topografía fue desarrollado a pleni- 
tud, permitiendo el desarrollo de habilidades y destrezas en los estudiantes de la carrera de Ingeniería Agropecuaria.

2. Las asignaturas de Construcciones Rurales, Edafología y Conservación de Suelos, Riego y Drenajes tienen estrecha relación con la asignatura de Topografía.

3. La existencia de un laboratorio de Topografía, equipado adecuadamente, mejorará la calidad del proceso de enseñanza y aprendizaje de esta materia académica.

4. Haber recibido en la asignatura de Topografía conocimientos teóricos y aplicaciones de programas informáticos y equipos.

A continuación, en la Tabla 6, se especifica la distribución de la encuesta por asignaturas.

TABLA 6. DISTRIBUCIÓN DE LA ENCUESTA POR ASIGNATURAS

\begin{tabular}{|c|c|c|}
\hline ASIGNATURA & MATRÍCULA & NO. DE ENCUESTADOS \\
\hline Construcciones rurales & 14 & 14 \\
\hline Topografía & 16 & 16 \\
\hline Edafología y conservación de suelos & 15 & 15 \\
\hline Riego y drenaje & 3 & 2 \\
\hline $\begin{array}{l}\text { Sistema de información geográfica } \\
\left({ }^{*}\right)\end{array}$ & 3 & 2 \\
\hline TOTAL & 51 & 49 \\
\hline
\end{tabular}

**- Matrícula total efectiva.

*- Se decidió incluir en la encuesta a los estudiantes de la asignatura de Sistemas de información geográficos al considerar que en ella se integran y aplican conocimientos, habilidades y destrezas adquiridos en la asignatura de Topografía.

ENCUESTAS DE OPINIÓN APLICADAS A LOS DOCENTES

La encuesta de opinión aplicada a los cinco docentes de las asignaturas en estudio, permite afirmar que:

1. Se requiere desarrollar habilidades y destrezas en los estudiantes de la carrera de Ingeniería Agropecuaria.

2. El componente práctico es fundamental para el desarrollo de habilidades y destrezas en los estudiantes de la carrera de Ingeniería Agropecuaria. 3.- La existencia de un laboratorio de Topografía, equipado adecuadamente, mejorará la calidad del proceso de enseñanza y aprendizaje de esta asignatura y de otras cuyos objetos de estudio sean similares. 4.- Los conocimientos, habilidades y destrezas instrumentales desarrolladas en la asignatura de Topografía son competencias profesionales que requiere el Ingeniero Agropecuario.

\section{GRADO DE DESARROLLO DE HABILIDADES Y DESTREZAS DEL CONOCIMIENTO QUE FUNDAMENTA LAS APLICACIONES DE LOS INSTRUMENTOS Y METODOLOGÍAS PARA LA SOLUCIÓN DE PROBLEMAS PRÁCTICOS DE LAS MATERIAS DE TOPOGRAFÍA, CONSTRUCCIONES RURALES, SUELOS Y DRENAJES}

Se aplicó un test de habilidades instrumentales topográficas a una muestra de 10 estudiantes, equivalente al $29 \%$ del total de estudiantes que cursaron y aprobaron la asignatura de Topografía, la que se considera representativa para un nivel de confianza del $95 \%$, con un error de muestreo de $26.43 \%$. Este, consistió en un ejercicio de medición de un área (polígono) representada por un sector del parqueadero de la UCSC utilizando instrumentos GPS (Spectra Precision, modelo promark). Luego se descargaron los datos registrados para representar gráficamente el resultado mediante el plano del levantamiento.

En la Tabla 7, se puede observar la selección aleatoria de los alumnos seleccionados para el desarrollo del test.

\begin{tabular}{|c|c|c|}
\hline MATERIA & $\begin{array}{l}\text { \# DE } \\
\text { ALUMNOS }\end{array}$ & $\begin{array}{l}\text { \# ALUMNOS } \\
\text { SELECCIONADOS }\end{array}$ \\
\hline Construcciones rurales & 14 & 3 \\
\hline Edafología y conservación de suelos & 15 & 3 \\
\hline Riego y drenaje & 3 & 2 \\
\hline Sistema de información geográfica & 3 & 2 \\
\hline SELECCIONADOS ALEATORIAMENTE & & 10 \\
\hline
\end{tabular}

A continuación, se muestran los resultados a los estudiantes evaluados, los cuales no tienen habilidades ni destrezas en el manejo instrumental de los equipos topográficos.

\section{DESCRIPCIÓN DEL TIPO DE PRÁCTICAS DE LABORATORIO QUE SON REQUERIDAS Y EL EQUIPAMIENTO NECESARIO PARA DESARROLLARLAS}

Para la determinación del tipo de prácticas a realizar y el equipamiento necesario para desarrollar habilidades y destrezas en los estudiantes que les permita la representación de la superficie terrestre, se aplicaron las siguientes técnicas: 1.-Encuesta a docentes y 2.-Entrevistas a expertos.

\section{PROPUESTA DE COMPETENCIAS GEOMÁTICAS DEL INGENIERO AGROPECUARIO}

1. Uso y aplicación de la cartografía, topográfica y temática (suelos, vegetación, clima, otras).

2. Uso y aplicación de instrumentos y métodos topográficos adecuados para la realización de levantamientos y replanteos con fines agropecuarios. 
3. Uso y aplicación de los fundamentos de Teledetección para la interpretación de la información registrada mediante sensores aerotransportados y satélites y su aplicación en la agricultura.

4. Implementación, gestión y aprovechamiento de Sistemas de Información Ceográfica (SIG) aplicados a la agricultura.

DESCRIPCIÓN DEL TIPO DE PRÁCTICAS DE LABORATORIO QUE SE REQUIEREN Y EL EQUIPAMIENTO NECESARIO PARA DESARROLLARLAS

Se determinaron un grupo de prácticas curriculares que permitirán desarrollar habilidades y destrezas para el manejo de equipos:

a. Teledetección y sus aplicaciones A la Agricultura: aborda contenidos referidos a las técnicas de captación de datos desde sensores remotos y su procesamiento visual-digital y aplicación al estudio de problemáticas presentes en el sector agrario y medio ambiental, así como las técnicas de GPS y radiométricas y su incorporación al concepto de agricultura de precisión.

b. Sistemas de Información Geográfica APlicados a la Agricultura: incluye contenidos referidos a la captación, entra$\mathrm{da}$, almacenaje, procesamiento de datos espaciales geo-referenciados en formatos ráster y vectorial, la representación cartográfica de los mismos, la asociación de bases de datos y el análisis de decisiones.

c. Topografía para Ingenieros AgroPECUARIOS: contenidos perfeccionados relativos a las características del relieve del terreno y a los métodos para su estudio y representación.

d. Cartografía tradicional y digital para Ingenieros Agropecuarios: contenidos relativos al conocimiento de los mapas, sus características, escalas, elementos, proyecciones y su elaboración, usando métodos tanto tradicionales como digitales.

e. Geo-estadística Agrícola: contenidos relacionados con la teoría de las variables regionalizadas, los semivariogramas, el análisis estructural, la validación cruzada, la geoestadística multivariada y sus aplicaciones en la caracterización de la variabilidad espacial de las propiedades agrícolas.
En la Tabla 8 se listan las asignaturas de la Malla de Ingeniería Agropecuaria del Rediseño Curricular (FETD-UCSG, 2016) que requieren de aplicaciones de conocimientos, habilidades y destrezas de las disciplinas geomáticas.

$\begin{array}{ll}\text { TABLA } & \text { 8. } \\
\text { COMPETENCIAS GEOMÁTICAS }\end{array}$
\begin{tabular}{ll} 
CICLO & ASIGNATURA \\
\hline 2 & Dibujo Técnico \\
\hline 4 & Meteorología Agrícola \\
\hline 4 & Topografía \\
\hline 5 & Construcciones Agropecuarias \\
\hline 5 & Sistemas de Información Geográfica \\
\hline 5 & Edafología \\
\hline 6 & Riego y Drenaje \\
\hline 7 & Agroforestería \\
\hline 7 & Sistemas de Producción Agrícolas: Ciclo corto \\
\hline 8 & Sistemas de Producción Agrícolas: Ciclo peremne \\
\hline 9 & Manejo de recursos naturales \\
\hline 10 & Trabajo de Titulación \\
\hline
\end{tabular}

Nota: Tomada de FETD-UCSG (2016).

PROPUESTAS DE ACTIVIDADES PRÁCTICAS Considerando el actual equipamiento disponible se propone un conjunto de actividades prácticas instrumentales para ambas asignaturas:

- Aplicación del programa AutoCAD como herramienta para el Dibujo Topográfico; lectura de mapas y planos convencionales; levantamiento de una poligonal cerrada; levantamiento topográfico con brújula, cinta métrica y clisímetro/ clinómetro; levantamiento y trazo por el método de deflexiones y conservación de azimut; levantamiento de dos poligonales, cálculo de áreas y orientación con dos puntos definidos sobre el terreno; entre otros.

- Se sugiere la elaboración de un Manual o Guía de Prácticas del Laboratorio, donde se desarrolle para cada práctica la siguiente información:

- Asignatura

- Objetivo General

- Resultados de Aprendizajes

- Contenidos

- Nombre de la Práctica

- Objetivo de la Práctica

- Equipamiento

- Síntesis del protocolo de ejecución

- Tiempo y Lugar (Campo/Gabinete)

- Bibliografía 


\section{LISTADO DE LOS ELEMENTOS QUE DEBEN SER TOMADOS EN CUENTA PARA EL DISEÑO DE UN LABORATORIO DE TOPOGRAFÍA, SOBRE LA BASE DE LOS RESULTADOS OBTENIDOS Y DE LA COMPARACIÓN CON OTROS LABORATORIOS QUE APARECEN EN LA LITERATURA CIENTÍFICA}

Para la determinación de los elementos que se requieren para el diseño del laboratorio de topografía se han considerado los resultados obtenidos en esta investigación. Estos son: a) Plan de Estudio de la Carrera de Ingeniería Agropecuaria; b) Misión del laboratorio de Geomática; y c) Infraestructura.

\section{DISCUSIÓN DE LOS RESULTADOS}

Los resultados de las encuestas y entrevistas permiten aceptar la hipótesis planteada, se prueba que la falta de un laboratorio de topografía con el equipamiento adecuado impide desarrollar las habilidades y destrezas instrumentales.

Este resultado confirma la opinión de los graduados de la carrera de Ingeniería Agropecuaria expresada en la encuesta aplicada a 24 graduados durante el año 2014 (UCSG - FEDT, 2014). El $56.5 \%$ de los estudiantes encuestados consideró que las prácticas académicas fueron suficientes para su formación profesional, el 57.9\% calificó de poco satisfactorios los laboratorios. Se acota que antes de este proyecto de investigación no existía laboratorio ni equipos topográficos en la FETD. Por tal motivo, resulta significativo que de los resultados obtenidos, el de mayor puntaje indique que la asignatura a mejorar en la carrea debe ser la de Topografía.

Los resultados alcanzados fundamentan las propuestas micro curriculares de las asignaturas de Topografía y de Sistemas de Información Geográficos. Las respectivas prácticas curriculares con uso de equipos de estas asignaturas y de otras materias que se consideren, deberán aplicar protocolos relacionados a las disciplinas en Geomática en sus actividades prácticas; lo que permitirá establecer un diseño de laboratorio de topografía más preciso.

\section{CONCLUSIONES Y RECOMENDACIÓN}

En conclusión, el tiempo de dedicación presencial de las asignaturas directamente relacionadas con aplicaciones para la representación de la superficie terrestre ocupa el $6.12 \%$ del total de horas presenciales curriculares. Los syllabus de las asignaturas involucradas en el proyecto no permiten establecer el tiempo del componente de las prácticas y los resultados de aprendizajes planificados para el desarrollo de destrezas instrumentales y aplicaciones de los conocimientos topográficos. La aplicación del test práctico aplicado a los estudiantes de la carrera de Ingeniería Agropecuaria evidencia que no tienen habilidades ni destrezas en el manejo instrumental del equipo topográfico. Se establecen las competencias profesionales que requiere el Ingeniero Agropecuario para la medición, representación e interpretación de la superficie terrestre.

Atendiendo a los resultados obtenidos, y con el laboratorio de Topografía en la FETD actualmente establecido, se recomienda al cuerpo docente reformar los syllabus para que puedan mejorar la carga práctica con la finalidad de incrementar la competitividad de los estudiantes en el mercado laboral.

\section{AGRADECIMIENTOS}

Agradecemos a la Universidad Católica de Santiago de Guayaquil (UCSG) y al Sistema de Investigación y Desarrollo (SINDE), por brindarnos las facilidades para el desarrollo de este tema de investigación.

\section{REFERENCIAS BIBLIOGRÁFICAS}

Consejo de Educación Superior (CES). (2014). Estructura de presentación para los proyectos de rediseño de la oferta académica vigente y nuevas ofertas a nivel de grado. Recuperado de http://www.ces.gob.ec/ doc/Plataforma-Postgrados/formato-proyectocarreras.pdf

FETD-UCSG. (2012). Syllabus de la Carrera de Ingeniería Agropecuaria, Malla Ajustada.

FETD-UCSG. (2014). Malla de Ingeniería Agropecuaria del Rediseño Curricular.

FETD-UCSG. (2016). Malla de Ingeniería Agropecuaria del Rediseño Curricular.

Ramírez, P. y Martínez, J. (2010). Concepciones de aprendizaje, creencias y conocimientos declarativos sobre la práctica profesional: Una aproximación con estudiantes de magisterio en educación preescolar. Medellín, Colombia: Institución Universitaria Tecnológico de Antioquía.

UCSG - FEDT. (2014). Informe de encuestas a graduados año 2014. Carreras Agropecuarias UCSG - FEDT: análisis e interpretación de datos 2015. 